\title{
An overview of directivity control methods of the parametric array loudspeaker
}

\author{
CHUANG SHI ${ }^{1}$, YOSHINOBU KAJIKAWA ${ }^{1}$ AND WOON-SENG GAN ${ }^{2}$
}

\begin{abstract}
A sound reproduction system usually consists of several types of loudspeakers to cater to sophisticated applications. The directivity of a loudspeaker is a measure of its efficiency in sending sounds to a particular direction instead of all directions. Demand to control the directivity of a sound reproduction system is gaining momentum with many new designs of directional loudspeakers, including the acoustic dome, horn loudspeaker, loudspeaker array, and parametric array loudspeaker (PAL). The PAL is an application of the parametric acoustic array in air, which generates a sound beam from the interaction of ultrasonic beams. The $P A L$ has several desired features, such as its narrow beamwidth over a wide frequency range, low sound attenuation over a long distance, and ability to reproduce perceptually near sound images. The PAL is also advantageous in using a smaller driving unit to transmit an equally narrow sound beam as compared to the conventional loudspeaker and broadside loudspeaker array. An overview of directivity control methods of the PAL is presented in this paper. In particular, acoustic models and signal processing techniques in controlling the directivity of the PAL are emphasized.
\end{abstract}

Keywords: Parametric array loudspeaker, Acoustic beamforming, Directivity model

Received 24 July 2014; Revised 4 December 2014; Accepted 4 December 2014

\section{INTRODUCTION}

The parametric array loudspeaker (PAL) is an application of the parametric acoustic array in air [1]. It was discovered by Westervelt in the early 1960s that two collimated high-frequency beams could result in the generation of a low-frequency sound beam as a result of nonlinear acoustic effects [2]. The high-frequency components were commonly referred to as the primary frequency and the low-frequency component generated from the interaction of primary frequencies was thus referred to as the difference frequency. After Westervelt published his discovery of the parametric acoustic array, Berktay studied a selfdemodulation phenomenon caused by the same nonlinear acoustic effects. Berktay found out that when an acoustic wave was transmitted in the form of a pulsed carrier, it was self-demodulated to its envelope, which consisted of much lower frequency components than the carrier frequency, after a sufficiently large propagation distance [3]. This was known as the Berktay's far-field solution. Therefore, the sound principle of the PAL happens when a modulated ultrasonic carrier is transmitted into air. The modulating

\footnotetext{
${ }^{1}$ Department of Electrical and Electronic Engineering, Kansai University, Osaka, Japan

${ }^{2}$ School of Electrical and Electronic Engineering, Nanyang Technological University, Singapore
}

Corresponding author:

C. Shi

Email: r148005@kansai-u.ac.jp sound is cumulatively formed in the beam of the ultrasonic carrier as described by the Berktay's far-field solution, and it will continue to propagate beyond the absorption distance where the ultrasonic carrier has been sufficiently attenuated [4].

Signal processing is of critical importance in the development of the PAL. Nonlinear distortions have been the top technical barrier to promote the use of the PAL. Ever since the PAL was invented [5], a variety of signal processing methods have been proposed to address nonlinear distortions in the PAL. Kamakura et al. have suggested that the square-root operation preprocess the envelope of the double sideband modulation method, which can substantially reduce the second harmonic distortion [6]. Kite et al. have further added the double integral operation in front of the square-root operation to treat the higher order harmonic distortions [7]. After that, the single sideband modulation method was introduced to fully eliminate harmonic distortions based on the Berktay's far-field solution. However, the practical performance of the single sideband modulation method was not as good as the double sideband modulation method. It was because that when pressure levels of primary frequencies were high, the Berktay's far-field solution was no longer valid [8]. Recently, there were many new methods applied in the PAL, such as the quadrature modulation method [9], recursive modulation method [10], and hybrid modulation method [11]. All of these methods were highly optimized to a specific acoustic model. They encountered the same limitation as the single 
sideband modulation method that discrepancies between the applied environment and acoustic model assumptions led to significant performance degradation. Therefore a superior method to address nonlinear distortions of the PAL should be able to adapt to the applied environment. One such solution is the nonlinear system identification of the PAL using a Volterra series [12-14]. On the other hand, a psychoacoustical method has been tested in the PAL to generate the virtual bass effect by equalizing the residual harmonic distortions that cannot be completely eliminated at this stage [15].

There are various studies that use the PAL as an accessible compact directional source. Sound field control is one of them targeting at tailored enhancement and attenuation of acoustic components [16]. The PAL has been employed in sound field control applications, such as the audio projection [17], three-dimensional sound reproduction [18], immersive communication [19], and active noise control (ANC) [20]. The computational complexity of a multichannel ANC system using PALs as secondary sources is greatly reduced, because crosstalk secondary path models can be removed $[21,22]$. Being relatively small in size, the PAL has substituted conventional miniature loudspeakers in the mobile phone [23] and interactive humanoid [24]. The PAL has also been employed in nondestructive testing, such as the landmine detection [25], weapon detection [26], material measurement [27], and art preservation [28].

Although a controllable sound beam enhances the ease of using the PAL in different applications, there has been only a limited number of studies to control the directivity of the PAL. The emitter of the PAL consists of numerous ultrasonic transducers. In the past studies, addressing nonlinear distortions of the PAL, the emitter of the PAL was considered as an ultrasonic source en bloc. However, when phased array techniques are applied to control the directivity of the PAL, ultrasonic transducers in the emitter of the PAL have to be configured into multiple channels, which are driven individually. This configuration can be interpreted as an array of PALs because every channel consists of a number of ultrasonic transducers and operates as a PAL. The delayand-sum beamformer has been proved effective in controlling the directivity of the PAL, but other sophisticated methods are usually not applicable due to lack of an accurate directivity model of the PAL with a low computational complexity [29].

The rest of this paper is organized as follows. Directivity control methods of the PAL are elaborated in Section II. The product directivity principle and past studies to improve the accuracy of the product directivity principle are presented in Section III(A). One of the latest directivity models of the PAL, namely the convolution model, is provided in Section III(B). One advantage of the convolution model is that environmental conditions, such as the room temperature and relative humidity, are taken into account. Using the convolution model, the beamwidth of the PAL is shown under different environmental conditions in Section IV(A). The constant beamwidth beamformer and digital beamsteerer of the PAL are described in Sections IV(B) and

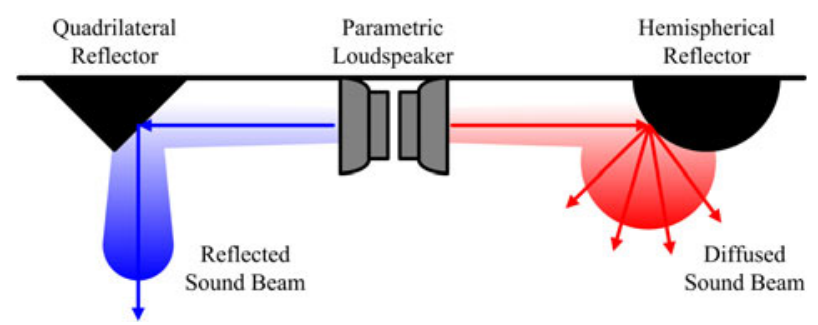

Fig. 1. Sketches of the quadrilateral and hemispherical reflectors for controlling the directivity of the PAL.

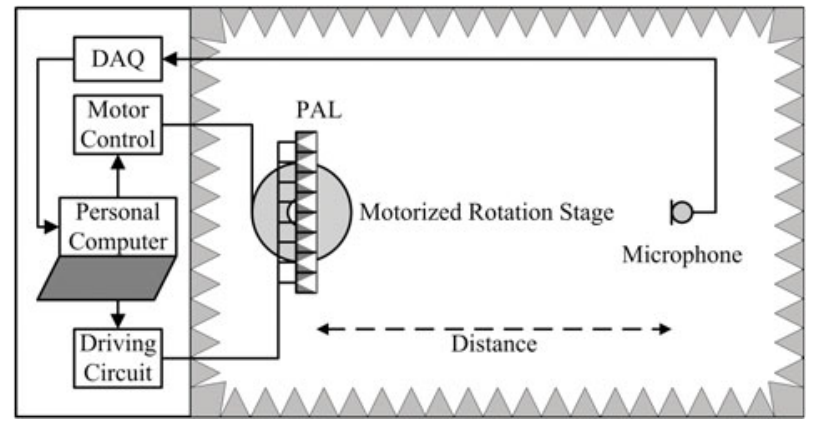

Fig. 2. Directivity measurement setup of the PAL.

IV(C). In addition, the digital beamsteerer of the PAL is examined when spatial aliasing at the primary frequency occurs. Lastly, Section V concludes this paper and charts new research directions of the PAL.

\section{DIRECTIVITY CONTROL METHOD DF THE PAL}

There are two types of directivity control methods of the PAL. They are the mechanical and signal processing methods. The mechanical method is built with motorized rotation stages and reflective objects, whereas the signal processing method is implemented in analog circuits and digital processors. The disadvantage of the mechanical method owes to its overall size and additional reflections caused by the motorized rotation stages and the reflective objects.

\section{A) Mechanical method}

The PAL is commonly used in the direct mode to project a sound beam to a listener. However, there is another reflected mode of the PAL. A virtual sound source is formed at the point of reflection when the PAL is placed to face a reflective surface as shown in Fig. 1. The PAL operating in the reflected mode has been employed in an ANC system [30], where the secondary source was invisible to the control point. Changes to the reflective surface can lead to certain directivity control of the PAL. The quadrilateral reflector diverts the beam direction of the PAL and the hemispherical reflector widens the beamwidth of the PAL [31]. Olszewski and Linhard [32] have reported that the presence of a listener's head in the near field of the PAL results in a diffused 


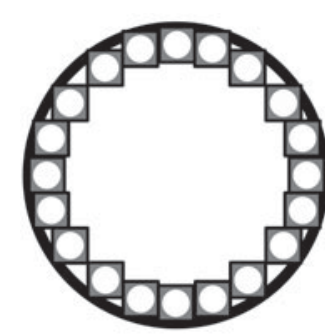

(a)

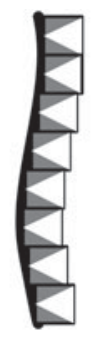

(b)

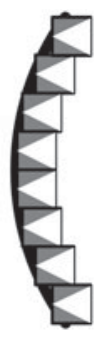

(c)

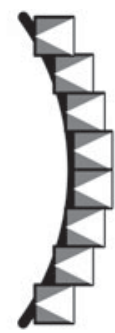

(d)

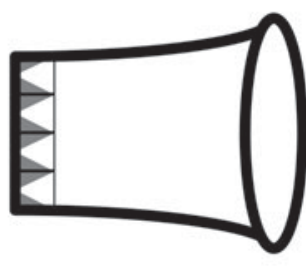

(e)

Fig. 3. Various array configurations of the PAL.

sound field. This observation can be explained by the similarity in shape between the listener's head and hemispherical reflector.

A motorized rotation stage can easily change the beam direction of the PAL. Ishii et al. [33] developed an indoor navigation system, in which the PAL was installed on a motorized rotation stage to send private messages to a moving listener. A similar setup is often adopted in the directivity measurement of the PAL. As illustrated in Fig. 2, the PAL is fastened on a motorized rotation stage and a microphone is placed in the far field of the PAL. Sound absorbing treatments to the walls of the measurement room are highly recommended. Moreover, an array of PALs can be tilted simultaneously by many motorized rotation stages. A prototype of one such motorized PAL array was built by Olszewski et al. [34], which consisted of four PALs tilting in unison. In this motorized PAL array, digital delays were also implemented in all the four channels to compensate for phase deviations among the PALs.

\section{B) Array configuration}

Array configuration is another important aspect of the directivity control of the PAL. Olszewski and Linhard [35] compared different configurations of the PAL and recommended a ring configuration that could narrow the beamwidth of the PAL over the low-frequency range. Figure 3(a) shows the front view of the ring configuration. Besides, a dedicated uneven surface as shown in Fig. 3(b) compensated for phase deviations among ultrasonic transducers [34]. A curved PAL mimicking the concave lens as shown in Fig. 3(c) was used in global ANC [36]. The focus point of the concave PAL was configured at the noise source to create an exact anti-noise source to reduce the noise level globally. In the three-dimensional sound reproduction, a convex PAL as shown in Fig. 3 (d) outperformed the concave PAL in terms of the size of the sweet spot [37]. Besides changing the array configuration of the PAL, the directivity of the PAL was also narrowed when a horn was attached to the PAL, which was shown in Fig. 3(e) [38].

An array of PALs was used as a multi-beam PAL and was mounted on the ceiling of a conference room to form a corresponding number of personal audio zones simultaneously [39]. As compared to the loudspeaker array, the power consumption and control complexity of this multibeam PAL were reduced significantly. In a mixed reality system, an icosahedral PAL transmitting 10 sound beams were employed to create virtual sound sources to provide a user with immersive listening experience [40]. The icosahedral PAL outperformed small-scale loudspeaker systems in terms of its localization accuracy, because the sound field reproduced by the icosahedral PAL was independent from individual head-related transfer functions.

In the acoustic measurement of a reverberant environment, a diffused acoustic source is essential but very expensive. An omnidirectional PAL was implemented by pinning hundreds of ultrasonic transducers on hollow sphere foam [41]. This omnidirectional PAL became a budget substitute to the expensive dodecahedron loudspeaker and demonstrated a more stable omnidirectional pattern over a wide frequency range. The low-frequency sound level of this omnidirectional PAL was relatively soft, which was another recognized drawback of the PAL [15].

\section{C) Array signal processing}

Figure 4 shows a delay-and-sum beamforming structure of the steerable PAL [42]. The weight for the $m$ th channel is denoted as $w_{m}$, where $m=0,1,2, \ldots, M-1$ and $M$ is the number of channels in the steerable PAL. Usually, the same delays are applied to all primary frequencies in every channel, which are given by

$$
\tau_{m}=m \frac{d}{c_{0}} \sin \theta_{0}
$$

where $d$ is the uniform spacing between neighboring channels; $c_{0}$ is the speed of sound; $\theta_{0}$ is the steering angle [43]. When the wavefront of the primary frequency is formed in one direction, the difference frequency generated from the interaction of primary frequencies is strengthened in the same direction. The quantitative description of this statement is called the product directivity principle, which is elaborated in the next section.

The effectiveness of the steerable PAL was validated in an application of ANC [44]. It was noted that when the PAL was used as the secondary source, not only was the noise level at the control point reduced, spillovers of the antinoise wave to other areas were also restricted. Moreover, the steerable PAL enabled the relocation of the control point to follow a moving listener. In a field programmable gate array (FPGA) implementation of the steerable PAL [45], 


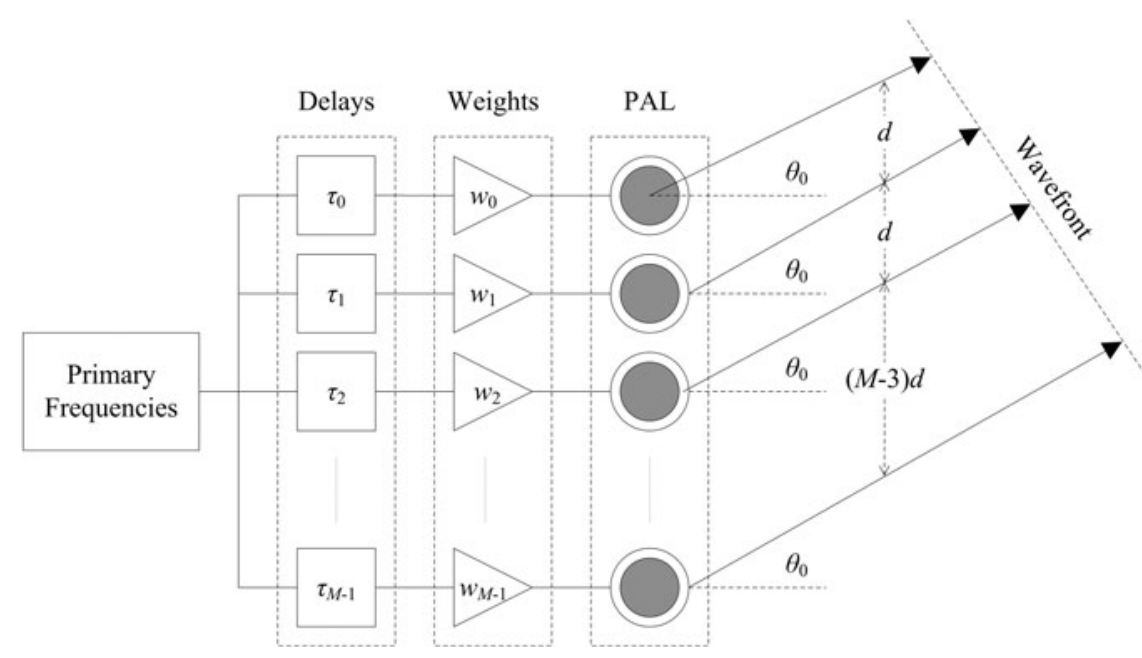

Fig. 4. Delay-and-sum beamforming structure of the steerable PAL.

fractional delay filters provided arbitrary delays for the primary frequency and consequently improved the angular resolution that was limited by the sampling interval. Instead, another FPGA implementation of the steerable PAL had to adopt a sampling rate at $1.4 \mathrm{MHz}$ to yield an angular resolution of $2^{\circ}$ [46]. A remarkable advantage of the steerable PAL in [46] was that it consisted of 576 channels and could transmit multiple controllable sound beams simultaneously.

The steerable PAL is gaining attention in assistive hearing too. For example, an elderly person with a hearing loss is watching a television program together with other people with no hearing loss. The steerable PAL can supplement the television loudspeaker system to enhance the listening experience of the elderly person with a hearing loss and prevent the excessive sound from spreading to the other people [47]. Furthermore, an array of PALs can reproduce an arbitrary wavefront with the help of wave field synthesis (WFS) techniques. A global quiet zone can be created regardless of the listener's position when virtual anti-noise sources are created in the vicinity of all actual noise sources [48].

\section{DIRECTIVITY MODEL DF THE PAL}

In this section, directivity models of the PAL are elaborated. The directivity of the PAL plays an important role in various applications of the PAL. Although there have been many nonlinear acoustic models describing the sound principle of the PAL, they seldom address the directivity of the PAL. The most widely used nonlinear acoustic models including the Westervelt and Khokhlov-Zabolotskaya-Kuznetsov (KZK) equations [2-4] have no general analytical solution. Numerical solutions are only available with a heavy computational load, especially when the directivity of the PAL is computed over a wide angle range. Therefore, it has been a long existing problem to accurately compute the directivity of the PAL with a low computational complexity when directivities of primary frequencies are given.

\section{A) Product directivity principle}

Early attempts to model the directivity of the PAL were carried out on the basis of the product directivity principle [49]. If the emitter of the PAL was a Gaussian source en bloc, the product directivity of two primary frequencies was able to approximate the directivity of the difference frequency. The product directivity principle is easily applied in the development of the steerable PAL. However, since assumptions of Gaussian source are partially ignored, the accuracy of the product directivity principle is not satisfactory.

To improve the accuracy of the product directivity principle, Shi and Gan [50] have introduced the equivalent Gaussian source array. In the equivalent Gaussian source array, the directivity of every Gaussian source approximates a lobe in the directivity of a primary frequency. Coefficients of the equivalent Gaussian the source array are determined by the nonlinear regression analysis based on the following model:

$$
D(\theta)=\sum_{n=1}^{N} p_{n} \exp \left[-\left(\frac{\omega a_{n}}{2 c_{0}}\right)^{2} \tan ^{2}\left(\theta-\theta_{n}\right)\right]
$$

where $\theta$ is the independent variable; $\omega$ is the primary frequency; $N$ is the number of Gaussian sources; $p_{n}, a_{n}$, and $\theta_{n}$ are the amplitude, radius, and steering angle of the $n$th Gaussian source, respectively. Measured directivities of the primary frequency provide the observational data in the nonlinear regression analysis. The use of the equivalent Gaussian source array allows assumptions of the Gaussian source to be valid in the PAL.

Based on the equivalent Gaussian source array, three modifications to the product directivity principle have been derived. They are the advanced product directivity (APD), exponential product directivity (EPD), and combined product directivity (CPD) models [51]. Taking the APD model into account, the directivity of the difference frequency is 
computed by

$$
\begin{aligned}
D_{d}(\theta)= & \sum_{m=1}^{N_{1}} \sum_{n=1}^{N_{2}}\left\{\frac{p_{1, m} p_{2, n} a_{1, m}^{2} a_{2, n}^{2}}{a_{1, m}^{2}+a_{2, n}^{2}}\right. \\
& \left.\times \exp \left[\frac{-a_{2, n}^{2} \omega_{2}^{2} \tan ^{2}\left(\theta-\theta_{2, n}\right)}{4 c_{0}^{2}}\right]\right\} .
\end{aligned}
$$

In equation (3), $N_{1}$ and $N_{2}$ are numbers of Gaussian sources of the two primary frequencies, respectively. The product directivity principle is applied to every pair of Gaussian sources in the equivalent Gaussian source array. Superposition of all the product directivities yields the directivity of the difference frequency. In the experiment validation in [51], the APD model has demonstrated a higher accuracy as compared to the product directivity principle, and the EPD and CPD models have shown similar accuracies but with higher computational complexities as compared to the APD model.

So far, there are two remaining drawbacks of the product directivity principle and models. Firstly, the beamwidth of the PAL is wider in practice than model results. Secondly, sidelobes of the PAL are observed in model results but are rarely found in practice. Therefore, a postprocessing method has been used for the product directivity principle and models. In this postprocessing method, the spline interpolation between local maxima of the model result leads to an improved match to the measured directivity of the difference frequency $[52,53]$. Nevertheless, this postprocessing method lacks theoretical evidences.

\section{B) Convolution model}

Recently, a convolution model based on the Westervelt's directivity has been originated. The convolution model provides an accurate directivity model for the PAL. Derivation of the convolution model is presented in this subsection and three applications of the convolution model are provided in the Section IV.

Firstly, according to assumptions made by Westervelt [2], primary frequency beams are extremely narrow and perfectly collimated so that a one-dimensional equation is adequate to represent the source distribution. The complex form of the primary source pressure level is assumed as

$$
p(x, t)=\sum_{m=1}^{2} P_{m} \exp \left[-\alpha_{m} x+i \omega_{m}\left(t-\frac{x}{c_{0}}\right)\right],
$$

where $P_{m}$ and $\alpha_{m}$ are the amplitude and attenuation rates of the $m$ th primary frequency at $\omega_{m}$, respectively; and $i$ is the imaginary unit.
The source strength density of the primary source is known as

$$
q(x, t)=\frac{\beta}{\rho_{0}^{2} c_{0}^{4}} \frac{\partial^{2}}{\partial t} p^{2}(x, t),
$$

where $\beta$ is the nonlinear coefficient and $\rho_{0}$ is the density of air. Hence, when equation (4) is inserted into equation (5), the source strength density of the difference frequency is obtained as

$$
\begin{aligned}
q_{d}(x, t)= & \frac{\beta P_{1} P_{2}}{\rho_{0}^{2} c_{0}^{4}}\left(-i \omega_{d}\right) \\
& \times \exp \left[-\left(\alpha_{1}+\alpha_{2}\right) x-i \omega_{d}\left(t-\frac{x}{c_{0}}\right)\right],
\end{aligned}
$$

where $\omega_{d}$ is the difference frequency, much lower than primary frequencies.

The geometry in Fig. 5 is applied to derive the Westervelt's directivity, which yields the pressure level of the difference frequency in the form of

$$
\begin{aligned}
p_{d}\left(R_{0}, \theta\right)= & \frac{\beta P_{1} P_{2} \omega_{d}^{2}}{4 \pi \rho_{0} c_{0}^{4} R_{0}\left(\alpha_{1}+\alpha_{2}\right)}\left[-1+i \frac{\omega_{d} \tan ^{2} \theta}{2 c_{0}\left(\alpha_{1}+\alpha_{2}\right)}\right]^{-1} \\
& \times \exp \left[-i \omega_{d}\left(t-\frac{R_{0}}{c_{0}}\right)\right]
\end{aligned}
$$

where $R_{0}$ is the distance from the observation point to the PAL; and $R$ is distance from the observation point to the virtual source located at $x$. The derivation of equation (7) follows the same steps of equation (12) in [2], but the approximation of $R_{0}-R \approx x-x \frac{\tan ^{2} \theta}{2}$ is adopted here instead of the one of $R_{0}-R \approx x \cos \theta$ used in [2]. Therefore, the Westervelt's directivity is given by the absolute value of the second term of equation ( 7 ), i.e.

$$
D_{W}(\theta)=\frac{1}{\sqrt{1+\frac{\omega_{d}^{2} \tan ^{4} \theta}{4 c_{0}^{2}\left(\alpha_{1}+\alpha_{2}\right)^{2}}}} .
$$

Using the Westervelt's directivity in equation (8), the magnitude of equation ( 7 ) can be rewritten as

$$
\left|p_{d}\left(R_{0}, \theta\right)\right|=\frac{\beta P_{1} P_{2} \omega_{d}^{2}}{4 \pi \rho_{0} c_{0}^{4} R_{0}\left(\alpha_{1}+\alpha_{2}\right)} D_{W}(\theta) .
$$

When collimated primary frequency beams are transmitted at an angle of $\theta_{0}$, they can be expressed in directivities as

$$
D_{1}(\theta)=P_{1} \delta\left(\theta-\theta_{0}\right)
$$

and

$$
D_{2}(\theta)=P_{2} \delta\left(\theta-\theta_{0}\right),
$$

where $\delta(x)$ is the Kronecker delta function that gives 1 when $x=0$ and o when $x \neq 0$. Hence, the pressure level of the difference frequency is obtained by rotating the coordinate system of equation (9), i.e.

$$
\left|p_{d}\left(R_{0}, \theta\right)\right|=\frac{\beta D_{1}\left(\theta_{0}\right) D_{2}\left(\theta_{0}\right) \omega_{d}^{2}}{4 \pi \rho_{0} c_{0}^{4} R_{0}\left(\alpha_{1}+\alpha_{2}\right)} D_{W}\left(\theta-\theta_{0}\right) .
$$

Under the assumption of the weak nonlinear interaction, the superposition principle of sound waves is still valid in 


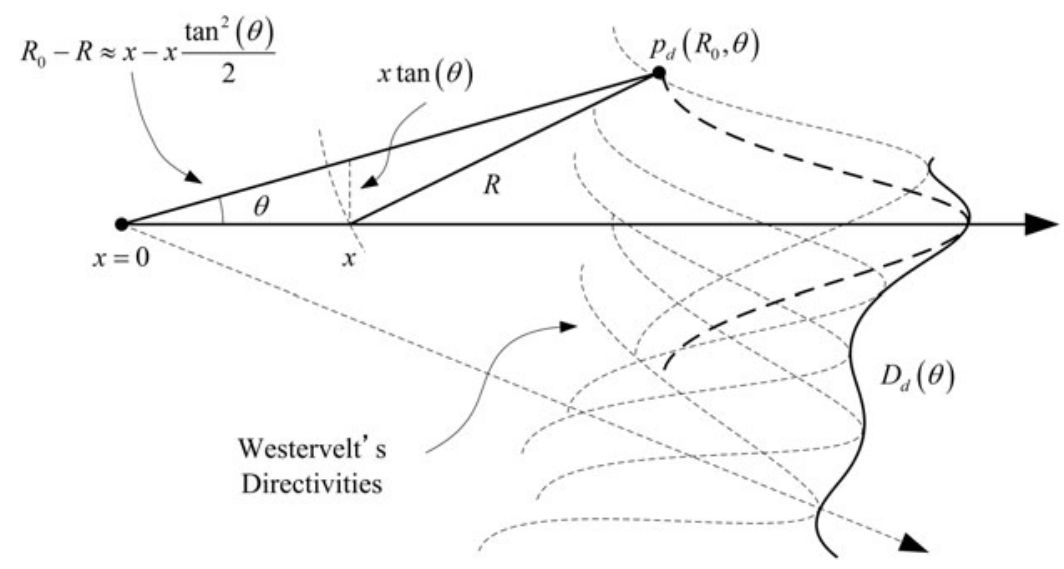

Fig. 5. Illustration of the Westervelt's directivity and convolution model of the PAL.

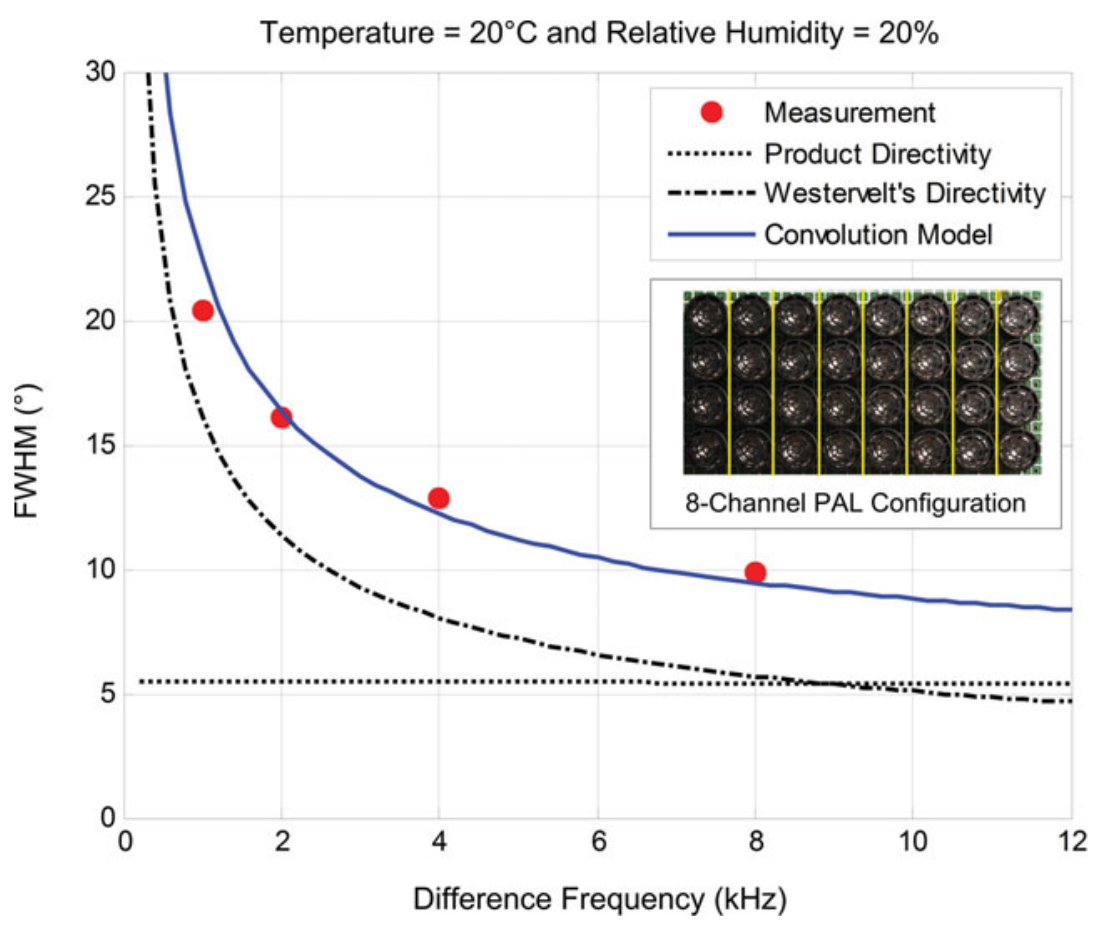

Fig. 6. An experimental validation of the convolution model.

the PAL. When a few pairs of collimated primary frequency beams are transmitted at different angles, the pressure level of the difference frequency is expressed by a summation as

$$
\begin{aligned}
\left|p_{d}\left(R_{0}, \theta\right)\right|= & \frac{\beta \omega_{d}^{2}}{4 \pi \rho_{0} c_{0}^{4} R_{0}\left(\alpha_{1}+\alpha_{2}\right)} \\
& \times \sum_{n=1}^{N} D_{1}\left(\theta_{n}\right) D_{2}\left(\theta_{n}\right) D_{W}\left(\theta-\theta_{n}\right),
\end{aligned}
$$

where $D_{1}\left(\theta_{n}\right)$ and $D_{2}\left(\theta_{n}\right)$ are equal to pressure levels of primary frequencies transmitted at the angle of $\theta_{n}$ when $n=1,2, \ldots, N$ and $N$ is the number of collimated beam pairs. In the directivity measurement, the directivity of a sound source is measured at a fixed distance from the source location and only at discrete angles. In this case, $N$ becomes the number of angles where the directivity measurement is taken.
Based on equation (13), the directivity of the difference frequency is written as

$$
D_{d}(\theta)=\sum_{n=1}^{N} D_{1}\left(\theta_{n}\right) D_{2}\left(\theta_{n}\right) D_{W}\left(\theta-\theta_{n}\right) .
$$

It is noted that equation (14) is in the form of a discrete convolution, which can be rewritten as

$$
D_{d}(\theta)=\left[D_{1}(\theta) D_{2}(\theta)\right] \otimes D_{W}(\theta),
$$

where $\otimes$ denotes the discrete convolution operation. In addition, when the PAL adopts the beamforming structure shown in Fig. 4, theoretical directivities of primary frequencies are computed by

$$
D_{1,2}(\theta)=\left|\sum_{m=1}^{M} \bar{w}_{m} \exp \left[i \frac{w_{1,2}}{c_{0}} m d\left(\sin \theta-\sin \theta_{0}\right)\right]\right|,
$$


where $\bar{w}_{m}=\frac{w_{m}}{\sum_{m=1}^{M}\left|w_{m}\right| \mid}$ is the normalized weight for the $m$ th channel.

In equation (15), the convolution of the product directivity with the Westervelt's directivity yields the directivity of the difference frequency. Thus, it is named as the convolution model. Shi and Kajikawa [54] have reported some experiment validations of the convolution model. Here another experiment validation is presented. A picture of the PAL consisting of 8 channels is shown in Fig. 6. When the steering angle is set to $0^{\circ}$, all the channels of this PAL are driven by the same modulated carrier. The full-width at half maximum (FWHM) of the beam is measured in directivities of the difference frequency at $1,2,4$, and $8 \mathrm{kHz}$. Measurement results are compared with the model results of the product directivity principle, Westervelt's directivity, and convolution model. Figure 6 shows that the convolution model has demonstrated the closest match to measurement results.

\section{DIRECTIVITY DESIGN OF THE PAL}

The convolution model in equation (15) has opened an opportunity to directivity control methods of the PAL. In the past studies, there have been some attempts to apply sophisticated array signal processing methods in the PAL $[49,55]$. But they have been hindered owing to lack of an effective directivity model. Using the convolution model, we are able to study directivity control methods that were difficult to investigate. Therefore, three applications of the convolution model in the PAL are elaborated in this section.

\section{A) Environmental influences on the FWHM of the PAL}

The temperature and relative humidity are two of the affecting factors in nonlinear acoustic effects in air. Their influence on the directivity of the PAL has yet to be addressed. The convolution model based on the Westervelt's directivity is a function of the difference frequency, speed of sound, and attenuation rates of primary frequencies. The temperature and relative humidity determine the speed of sound together with attenuation rates of primary frequencies. Hence, it is interesting to see how the temperature and relative humidity affect the directivity of the PAL. A PAL consisting of 8 equally spaced channels is used in this study. The interchannel spacing and steering angle are set to $1 \mathrm{~cm}$ and $0^{\circ}$, respectively. The difference frequency is generated as a sine sweep from $500 \mathrm{~Hz}$ to $12 \mathrm{kHz}$, which has the same typical frequency range as one commercial unit of the PAL [11]. The FWHM is measured in the directivity of the PAL.

In Fig. $7(a)$, the relative humidity is set to $50 \%$, while the temperature varies from $0^{\circ} \mathrm{C}$ to $30^{\circ} \mathrm{C}$. Higher difference frequencies and lower temperatures result in narrower beamwidths of the PAL. This observation is similar to that made in the broadside loudspeaker array. The directivity of
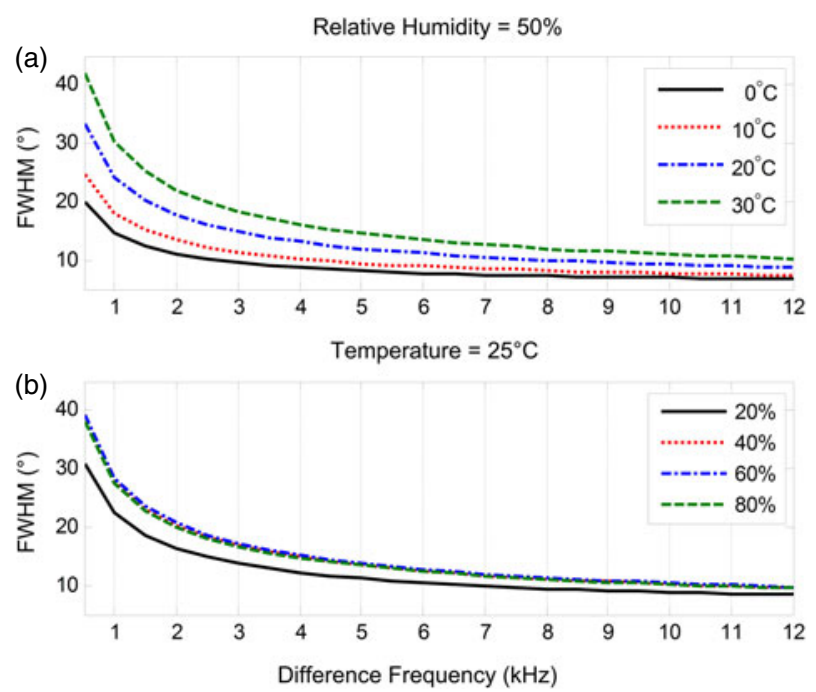

Fig. 7. FWHM of the PAL changing with the temperature and relative humidity.

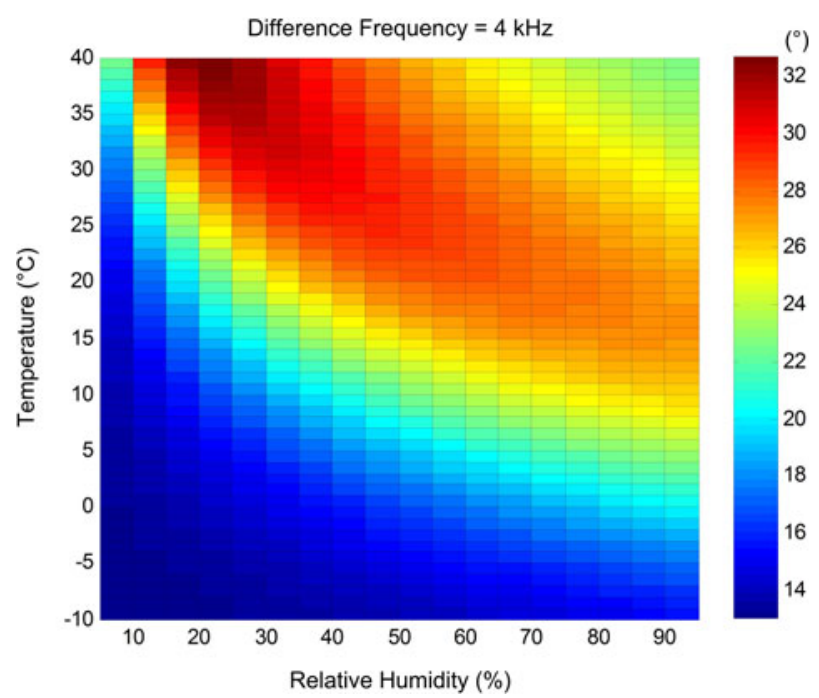

Fig. 8. FWHM of the PAL under different environmental conditions when the difference frequency is transmitted at $4 \mathrm{kHz}$.

a broadside loudspeaker array depends on the wavenumber. As the speed of the sound is slow at a lower temperature, both a higher frequency and a lower temperature lead to a larger wavenumber. Therefore, a narrow beamwidth of the broadside loudspeaker array is expected when the frequency is high and the temperature is low. In Fig. $7(\mathrm{~b})$, the temperature is set to $25^{\circ} \mathrm{C}$, while the relative humidity varies from $20 \%$ to $80 \%$. A unique observation of the PAL can be made in Fig. 7 (b). The beamwidth of the PAL is almost constant regardless of the varying relative humidity in the range of $40-80 \%$. When the relative humidity is $20 \%$, the beamwidth of the PAL becomes much narrower because attenuation rates of primary frequencies decrease to a much smaller level [56].

Figure 8 shows the FWHM of the PAL when the difference frequency is fixed at $4 \mathrm{kHz}$. The temperature varies from $-10^{\circ} \mathrm{C}$ to $30^{\circ} \mathrm{C}$ and the relative humidity varies from $5 \%$ to $95 \%$. When the air is extremely dry and cold, the beamwidth of the PAL is narrow and independent from the 
(a)

Product Directivity Principle

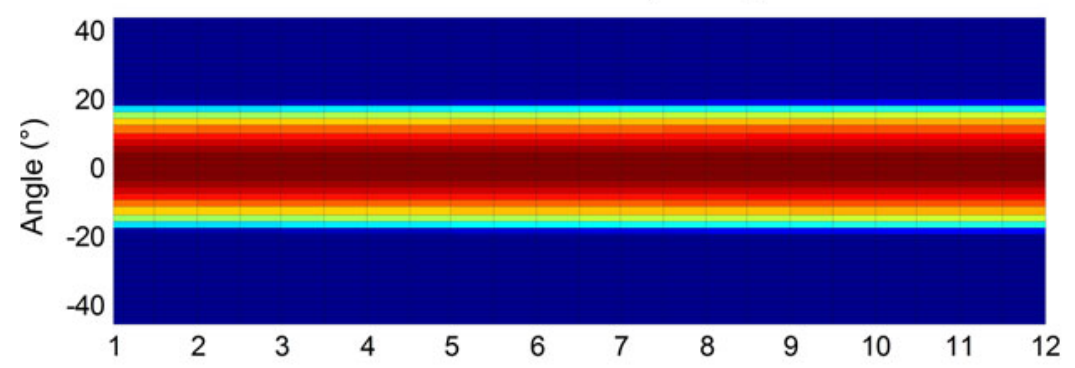

(b)

Convolution Model

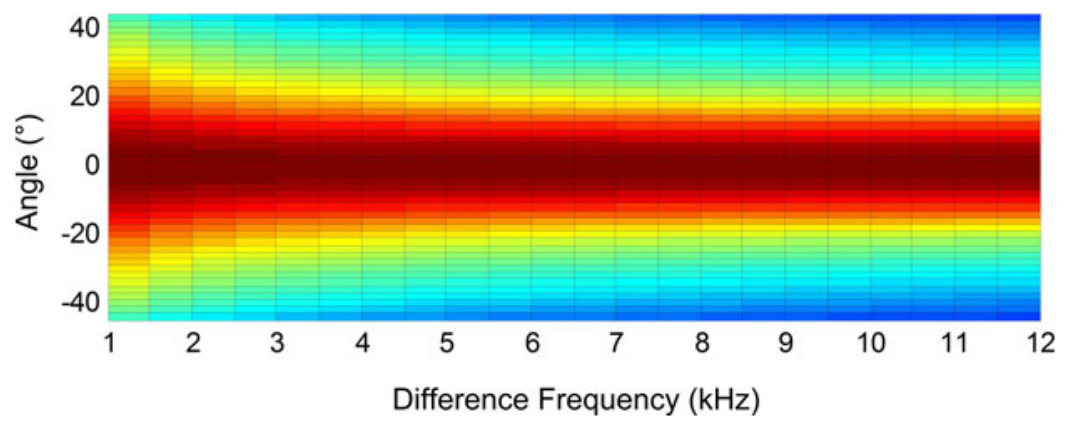

Fig. 9. Directivity of the PAL using the constant beamwidth beamformer.

temperature and relative humidity. This is because when the air is extremely dry and cold, attenuation rates of the primary frequencies are so low that the Westervelt's directivity becomes as sharp as the Dirac Delta function. Hence, the convolution model is simplified to the product directivity principle. As aforementioned, the product directivity principle tends to have a narrow beamwidth. On the other hand, when the temperature is high, the beamwidth of the PAL is relatively wide. We can conclude from Fig. 8 that the beamwidth of the PAL is relatively narrow in the desert region and wide in the tropical region. This knowledge of difference in directivity is beneficial to applications of the PAL related to sound field control [17-22].

\section{B) Constant beamwidth beamformer of the PAL}

In signal processing, the design of a finite impulse response filter based on window functions is a mature and simple technique. Similarly, window functions can be used in the design of a uniform linear array (ULA). When coefficients of a window function are adopted as weights for the ULA, the frequency response of the window function gives the beampattern of the ULA. Hence, the wavenumber is also referred to as the spatial frequency.

The Dolph-Chebyshev window is famous for having the narrowest mainlobe when the sidelobe attenuation is given. Moreover, sidelobe levels of the Dolph-Chebyshev window are always equal. When weights for a ULA are designed based on the Dolph-Chebyshev window, the beamwidth of the ULA is decided by the sidelobe attenuation and vice versa. The emitter of the PAL can be configured into a ULA.
Therefore, the constant beamwidth beamforming approach has been proposed for the PAL taking into account the single sideband modulation method [55]. In the single sideband modulation, there are two primary frequencies that are referred to as the carrier and sideband frequencies, respectively. In the constant beamwidth beamformer of the $\mathrm{PAL}$, the sidelobe attenuation of the carrier frequency is determined by the given null to null beamwidth, and the sidelobe attenuation of the sideband frequency is adjusted to make the first sidelobe of the sideband frequency coincide with the first null of the carrier frequency. Based on the product directivity principle, the null to null beamwidth of the PAL is equal to the given null to null beamwidth over all the difference frequencies.

To examine the constant beamwidth beamformer using the convolution model, the PAL consisting of 8 channels is adopted again, of which the interchannel spacing is set to $0.5 \mathrm{~cm}$. This interchannel spacing is feasible only in the compact configuration $[44,45]$. The difference frequency is generated as a sine sweep from 1 to $12 \mathrm{kHz}$. The lower sideband modulation (LSB) method sets one primary frequency at $40 \mathrm{kHz}$ as the carrier frequency and decreases the other primary frequency from 39 to $28 \mathrm{kHz}$ as the sideband frequency. The null to null beamwidth of the PAL is given by $40^{\circ}$. The steering angle is set to $0^{\circ}$. The design of the constant beamwidth beamformer gives the weight for every channel. Directivities of the difference frequency are plotted in Figs. 9(a) and 9(b) using the product directivity principle and convolution model, respectively. The constant null to null beamwidth is easily observed in Fig. 9(a), but there are no nulls in the convolution model as a result of the convolution with the Westervelt's directivity. Since the convolution 


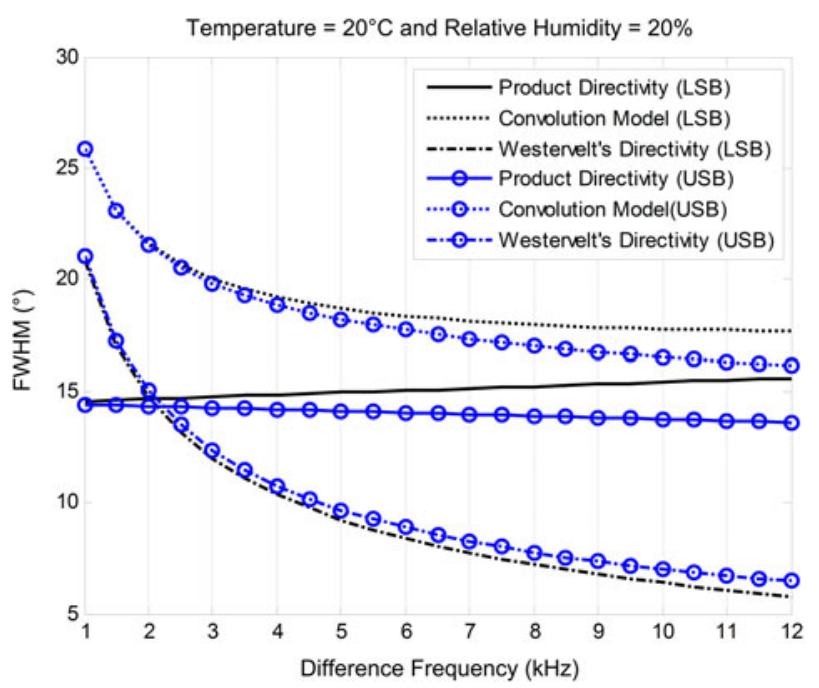

Fig. 10. FWHM of the PAL using the constant beamwidth beamformer.

model is proved more accurate than the product directivity principle, the FWHM is a more practical measure of the PAL's beamwidth rather than the null to null beamwidth.

In contrast to the LSB method, the upper sideband modulation (USB) method increases the sideband frequency from 41 to $52 \mathrm{kHz}$. FWHMs are measured in directivities of the PAL when the LSB and USB methods are adopted and results are shown in Fig. 10. There are several interesting observations. Firstly, the product directivity principle results in narrower FWHMs as compared to the convolution model. Secondly, when the difference frequency increases, the Westervelt's directivity becomes narrower and model results of the product directivity principle and convolution model become closer. Thirdly, the Westervelt's directivity plays a dominant role in the directivity of the PAL when the difference frequency is low. Lastly, the FWHM of the Westervelt's directivity exhibits an exponential decay against the difference frequency. The convolution model suggests that the product directivity of primary frequencies should be designed to have an exponential growth in the FWHM in order to implement an efficient constant beamwidth beamformer of the PAL.

\section{C) Digital beamsteerer of the PAL}

The digital signal is a discrete and discontinuous representation of information. The minimum integer delay in any digital system is determined by the sampling interval of this system. Based on equation (1), the available steering angle is consequently given by

$$
\theta_{n}=\sin ^{-1}\left(\frac{c_{0}}{d} n T_{s}\right)
$$

where $T_{s}$ is the sampling interval and $n$ is a natural number. When the interchannel spacing and sampling interval are set to $0.5 \mathrm{~cm}$ and $0.01 \mathrm{~ms}$, respectively, there is only one available steering angle existing at $43^{\circ}$. When the interchannel spacing is extended to $1 \mathrm{~cm}$, another steering angle becomes available at $20^{\circ}$, but spatial aliasing also occurs.
Derived from equation (17), there are three conventional solutions to the limited range of the steering angle, i.e. to implement the fractional delay filter [45], to use a high sampling rate [46], and to widen the interchannel spacing [50].

However, these solutions do not account for the sound principle of the PAL. Therefore, a dedicated digital beamsteerer has been specifically worked out for the PAL taking into account the single sideband modulation [49]. Different delays are applied to two primary frequencies, which are also referred to as the carrier and sideband frequencies. The carrier signal can be digitally generated with arbitrary delay so the carrier frequency is able to be steered to any direction. However, available steering angles of the sideband frequency remain limited by equation (17). Based on the product directivity principle, if the mainlobe of the sideband frequency is sufficiently wide, the directivity of the carrier frequency will contribute to the directivity of the PAL.

The PAL configuration remains the same as the previous subsection. The carrier and difference frequencies are transmitted at $40 \mathrm{kHz}$ and $4 \mathrm{kHz}$, respectively. Weights for the sideband frequency are designed by the ParksMcClellan algorithm [57]. When the steering angle is set to $20^{\circ}$, directivities of primary and difference frequencies are plotted in Figs. 11(a) and 11(c) when the LSB and USB methods are adopted, respectively. Based on the design of the digital beamsteerer, the mainlobe of the sideband frequency is much wider than the mainlobe of the carrier frequency. Therefore, the steering angle of the PAL is expected to depend on the directivity of the carrier frequency. Furthermore, when the steering angle varies from $-90^{\circ}$ to $90^{\circ}$, directivities of the difference frequency are plotted in Figs. 11(b) and 11(d). It can be observed that the digital beamsteerer is effective for both the LSB and USB methods until spatial aliasing occurs at large steering angles.

Because of the wide mainlobe of the sideband frequency, grating lobe elimination is not going to be effective in the digital beamsteerer of the PAL [50]. Spatial aliasing is thus the weakness of the digital beamsteerer. To illustrate this weakness, the interchannel spacing is extended to $1.5 \mathrm{~cm}$ and the rest of conditions are kept unchanged. Now the interchannel spacing is twice the wavelength of the sideband frequency at $44 \mathrm{kHz}$. When the steering angle is set to $20^{\circ}$, directivities of primary and difference frequencies are plotted in Figs. 12(a) and 12(c). As the grating lobe of the sideband frequency is as wide as the mainlobe, the difference in spatial periods of the carrier and sideband frequencies cannot separate all grating lobes sufficiently. Therefore, grating lobe elimination fails to occur. Once again, when the steering angle varies from $-90^{\circ}$ to $90^{\circ}$, directivities of the difference frequency are plotted in Figs. 12(b) and 12(c). Spatial aliasing is observed for all steering angles in the digital beamsteerer. It is confirmed that the digital beamsteerer requires a narrow interchannel spacing to avoid spatial aliasing at primary frequencies. Therefore, the compact configuration is of the utmost importance to the digital beamsteerer [49]. 

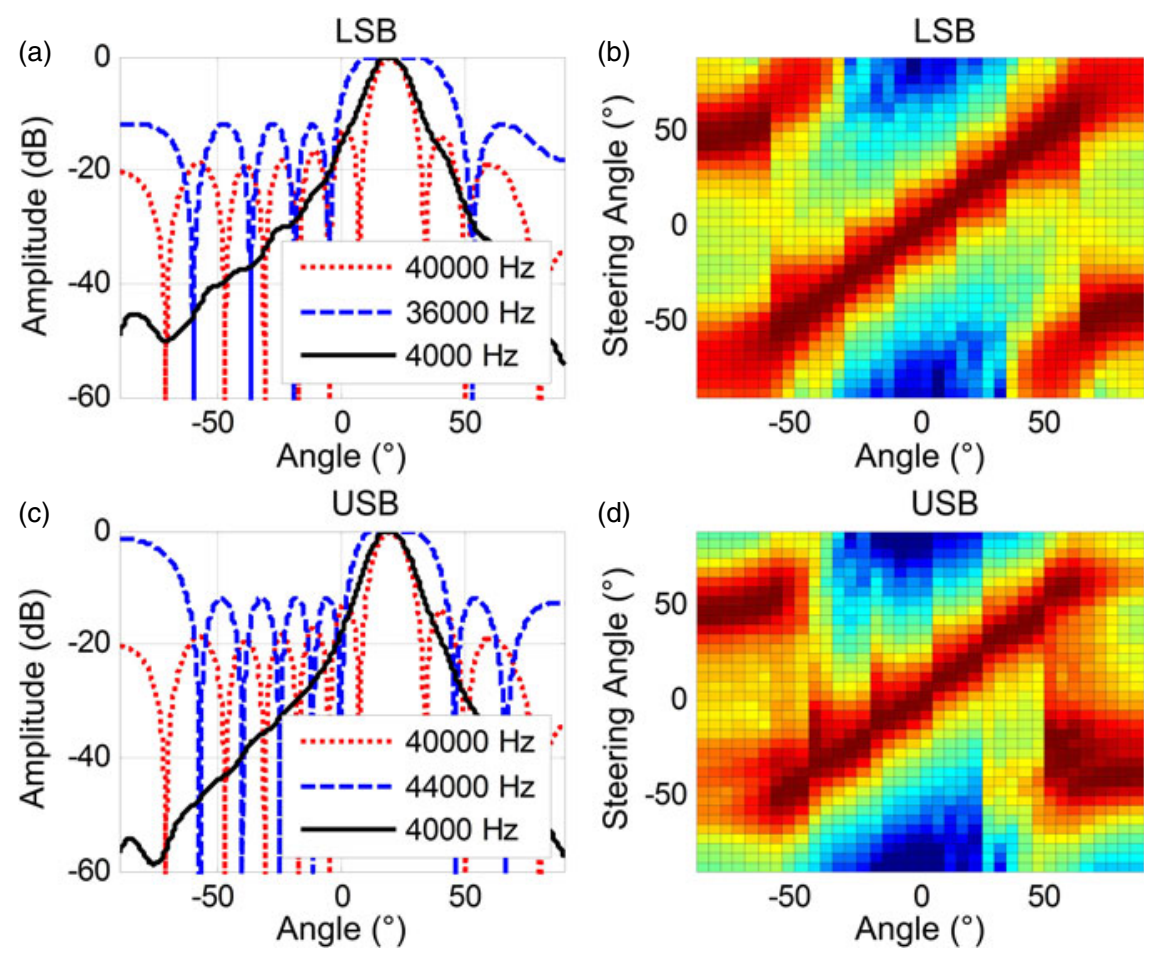

Fig. 11. Directivity of the PAL using the digital beamsteerer and compact configuration.
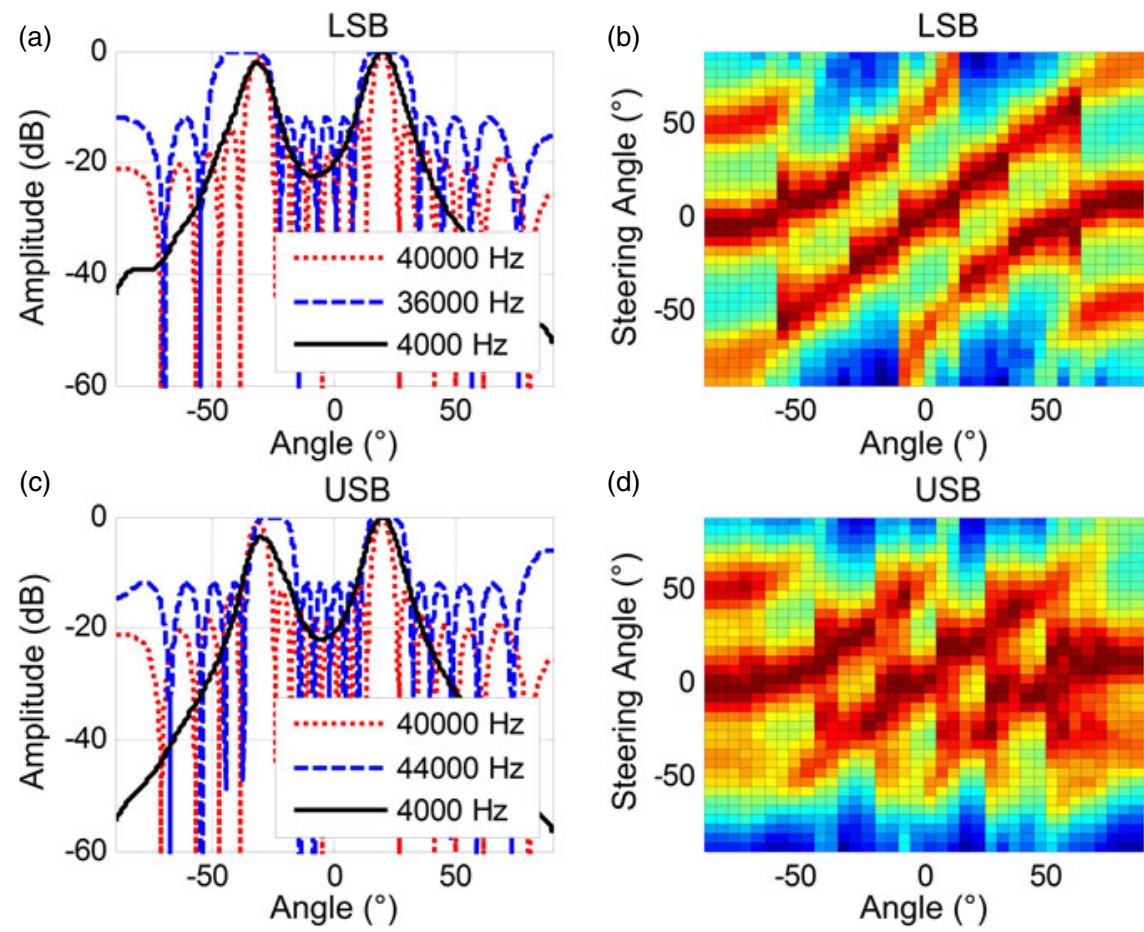

Fig. 12. Spatial aliasing example in the digital beamsteerer of the PAL.

\section{CONCLUSIONS}

This paper overviewed a wide range of directivity control methods of the PAL. The superb accuracy of the convolution model has been demonstrated through experiments. The convolution model has outperformed previous directivity models. Three applications of the convolution model in the directivity control of the PAL have been elaborated. The directivity of the PAL is found to be relatively narrow in desert regions and wide in tropical regions. The beamwidth of the PAL can double in a hot and humid climate than in a cold and dry climate. Environmental influences on the directivity of the PAL are of importance when the PAL is employed in sound field control. Furthermore, to develop 
the constant beamwidth beamformer of the PAL, it is suggested that the product directivity have an exponentially growing beamwidth to compensate for the window effect of the Westervelt's directivity in spatial domain. Nevertheless, the directivity of the PAL in the low-frequency band is dominated by the Westervelt's directivity and only mechanical methods are efficient to control it. Lastly, the digital beamsteerer of the PAL has been examined using the convolution model. It is found that the digital beamsteerer is sensitive to spatial aliasing at the primary frequency and thus the compact configuration is of the utmost importance to the digital beamsteerer of the PAL to avoid spatial aliasing.

The convolution model has created new opportunities in the research area of the PAL. There have been many worthwhile attempts to apply the WFS and other sophisticated array signal processing methods to control the directivity of the PAL. But most of them have not been successful due to lack of an efficient directivity model. Trial and error methods of placing PALs in a sound field control application will be improved by introducing the convolution model. We look forward to more exciting applications of the PAL, for example in a smart city where sound field control can be widely applied to reduce noise pollution. This will accelerate the popularity of the PAL. Moreover, as the PAL generates sounds from ultrasound, it is able to be integrated with other established ultrasonic techniques, such as the time reversal, medical imaging, and even parametric receiving array. This paper has thus presented an up to date and comprehensive reference for future works of the PAL.

\section{ACKNOWLEDGEMENTS}

This work is supported by MEXT-Supported Program for the Strategic Research Foundation at Private Universities, 2013-2017.

\section{REFERENCES}

[1] Bennett, M.B.; Blackstock, D.T.: Parametric array in air. J. Acoust. Soc. Am., 57 (1975), 562-568.

[2] Westervelt, P.J.: Parametric acoustic array. J. Acoust. Soc. Am., 35 (1963), 535-537.

[3] Berktay, H.O.: Possible exploitation of non-linear acoustics in underwater transmitting applications. J. Sound Vib., 2 (1965), 435-461.

[4] Shi, C.; Gan, W.S.: Development of a parametric loudspeaker: a novel directional sound generation technology. IEEE Potentials, 29 (2010), $20-24$.

[5] Yoneyama, M.; Fujimoto, J.; Kawamo, Y.; Sasabe, S.: The audio spotlight: an application of nonlinear interaction of sound waves to a new type of loudspeaker design. J. Acoust. Soc. Am., 73 (1983), 1532-1536.

[6] Kamakura, T.; Yoneyama, M.; Ikegaya, K.: Developments of parametric loudspeaker for practical use, Int. Symp. Nonlinear Acoustics, Kobe, Japan, 1984.

[7] Kite, T.D.; Post, J.T.; Hamilton, M.F.: Parametric array in air: distortion reduction by preprocessing, Int. Congress on Acoustics, Seattle, USA, 1998.
[8] Aoki, K.; Kamakura, T.; Kumamoto, Y.: Parametric loudspeaker: characteristics of acoustic field and suitable modulation of carrier ultrasound. Electron. Commun. Japan (Part III: Fundam. Electron. Sci.), 74 (1991), 76-82.

[9] Liew, Y.H.: Signal processing techniques for sound reproduction in parametric arrays, Master Thesis, Nanyang Technological University, Singapore, 2002.

[10] Croft, J.J.; Spencer, M.E.; Norris, J.O.: Modulator processing for a parametric speaker system, Patent US6584205, USA, 2003.

[11] Shi, C.; Gan, W.S.: A preprocessing method to increase high frequency response of a parametric loudspeaker, APSIPA Annual Summit and Conf., Kaohsiung, Taiwan, 2013.

[12] Ji, W.; Gan, W.S.: Identification of a parametric loudspeaker system using an adaptive Volterra filter. Appl. Acoust., 73 (2012), 1251-1262.

[13] Hatano, Y.; Shi, C.; Kajikawa, Y.: A study on linearization of nonlinear distortions in parametric array loudspeakers, Int. Workshop on Smart Info-Media Systems in Asia, Ho Chi Minh City, Vietnam, 2014.

[14] Mu, Y.; Ji, P.; Ji, W.; Wu, M.; Yang, J.: Modeling and compensation for the distortion of parametric loudspeakers using a one-dimension Volterra filter. IEEE/ACM Trans. Audio, Speech, Lang. Process., 22 (2014), 2169-2181.

[15] Shi, C.; Mu, H.; Gan, W.S.: A psychoacoustical preprocessing technique for virtual bass enhancement of the parametric loudspeaker, Int. Conf. Acoustics, Speech, and Signal Processing, Vancouver, Canada, 2013.

[16] Elliot, S.: Active control of sound fields: enhancing signals and attenuating noise, Audio Engineering Society Conf., Guildford, UK, 2013.

[17] Aoki, S.; Toba, M.; Tsujita, N.: Sound localization of stereo reproduction with parametric loudspeakers. Appl. Acoust., 73 (2012), 1289-1295.

[18] Shi, C.; Nomura, H.; Kamakura, T.; Gan, W.S.: Spatial aliasing effects in a steerable parametric loudspeaker for stereophonic sound reproduction. IEICE Trans. Fundam. Electron. Commun. Comput. Sci., E97-A (2014), 1859-1866.

[19] Gan, W.S.; Tan, E.L.; Kuo, S.M.: Audio projection. IEEE Signal Process. Mag., 28 (2011), 43-57.

[20] Lam, B.; Gan, W.S.; Shi, C.: Feasibility of a length-limited parametric source for active noise control applications, Int. Congress on Sound and Vibration, Beijing, China, 2014.

[21] Tanaka, K.; Shi, C.; Kajikawa, Y.: Study on active noise control system using parametric array loudspeakers, Forum Acusticum, Krakow, Poland, 2014.

[22] Tanaka, K.; Shi, C.; Kajikawa, Y.: Multi-channel active noise control using parametric array loudspeakers, APSIPA Annu. Summit and Conf., Siem Reap, Cambodia, 2014.

[23] Nakashima, Y.; Yoshimura, T.; Naka, N.; Ohya, T.: Prototype of mobile super directional loudspeaker. NTT DoCoMo Tech. J., 8 (2006), 25-32.

[24] Nakadai, K.; Tsujino, H.: Towards new human-humanoid communication: Listening during speaking by using ultrasonic directional speaker, IEEE Int. Conf. Robotics and Automation, Barcelona, Spain, 2005.

[25] Haupt, R.W.; Rolt, K.D.: Standoff acoustic laser technique to locate buried land mines. Linc. Lab. J., 15 (2005), 3-22.

[26] Achanta, A.; McKenna, M.; Heyman, J.: Non-linear acoustic concealed weapons detection, Applied Imagery and Pattern Recognition Workshop, Washington, USA, 2005.

[27] Kuang, Z.; Ye, C.; Wu, M.; Yang, J.: A method for measuring the absorption coefficient of materials in situ using a parametric loudspeaker, Int. Congress on Sound and Vibration, Cairo, Egypt, 2010. 
[28] Calicchia, P.; Simone, S.D.; Marcoberardino, L.D.; Marchal, J.: Nearto far-field characterization of a parametric loudspeaker and its application in non-destructive detection of detachments in panel paintings. Appl. Acoust., 73 (2012), 1296-1302.

[29] Shi, C.: Generation of a steerable audio beam from an ultrasonic transducer array. APSIPA Newsl., 7 (2014), 16-18.

[30] Tachi, R.; Tanaka, N.: Active noise control using acoustic wave reflection of parametric speaker, Asia Pacific Vibration Conf., Christchurch, New Zealand, 2009.

[31] Morise, M.; Ikefuji, D.; Tujii, H.; Hirokawa, K.; Nishiura, T.: A design of reflective audio spot with reflective objects, Int. Congress on Acoustics, Sydney, Australia, 2010.

[32] Olszewski, D.; Linhard, K.: Imperfections of parametrically generated sound beams caused by reflexions, Symp. Ultrasonic Electronics, Nagoya, Japan, 2006.

[33] Ishii, K.; Yamamoto, Y.; Imai, M.; Nakadai, K.: A navigation system using ultrasonic directional speaker with rotating base. Lecture Notes in Comput. Sci., 4558 (2007), 526-535.

[34] Olszewski, D.; Prasetyo, F.; Linhard, K.: Steerable highly directional audio beam loudspeaker, Interspeech, Lisbon, Portugal, 2005.

[35] Olszewski, D.; Linhard, K.: Optimum array configuration for parametric ultrasound loudspeakers using standard emitters, Interspeech, Pittsburgh, USA, 2006.

[36] Tanaka, N.; Tanaka, M.: Mathematically trivial control of sound using a parametric beam focusing source. J. Acoust. Soc. Am., 129 (2011), $165-172$.

[37] Ikefuji, D.; Tsuji, H.; Masnaga, S.; Nakayama, M.; Nishiura, T.; Yamashita, Y.: Reverberation steering and listening area expansion on 3-D sound field reproduction with parametric array loudspeaker, APSIPA Annu. Summit Conf., Siem Reap, Cambodia, 2014.

[38] Sayin, U.; Guasch, O.: Directivity control and efficiency of parametric loudspeakers with horns. J. Acoust. Soc. Am., 134 (2013), EL153-EL157.

[39] Olszewski, D.; Linhard, K.: Highly directional multi-beam audio loudspeaker, Interspeech, Pittsburgh, USA, 2006.

[40] Sugibayashi, Y.; Kurimoto, S.; Ikefuji, D.; Morise, M.; Nishiura, T.: Three-dimensional acoustic sound field reproduction based on hybrid combination of multiple parametric loudspeakers and electrodynamic subwoofer. Appl. Acoust., 73 (2012), 1282-1288.

[41] Sayin, U.; Artis, P.; Guasch, O.: Realization of an omnidirectional source of sound using parametric loudspeakers. J. Acoust. Soc. Am., 134 (2013), 1899-1907.

[42] Shi, C.; Gan, W.S.: Analysis and calibration of system errors in steerable parametric loudspeakers. Appl. Acoust., 73 (2012), 1263-1270.

[43] Shi, C.; Gan, W.S.: Steerable parametric loudspeaker with preprocessing methods, Int. Congress on Acoustics, Montreal, Canada, 2013.

[44] Tanaka, N.; Tanaka, M.: Active noise control using a steerable parametric array loudspeaker. J. Acoust. Soc. Am., 127 (2010), 3526-3537.

[45] Wu, S.; Wu, M.; Huang, C.; Yang, J.: FPGA-based implementation of steerable parametric loudspeaker using fractional delay filter. Appl. Acoust., 73 (2012), 1271-1281.

[46] Takeoka, S.; Yamasaki, Y.: Acoustic projector using directivity controllable parametric loudspeaker array, Int. Congress on Acoustics, Sydney, Australia, 2010.

[47] Shi, C.: Investigation of the steerable parametric loudspeaker based on phased array techniques. Doctoral Dissertation, Nanyang Technological University, Singapore, 2013.
[48] Ishimori, A.; Tanaka, N.; Iwamoto, H.: Global active noise control using a delayed driving parametric array loudspeaker. Trans. Japan Soc. Mech. Eng. Ser. C, 79 (2013), 604-616.

[49] Gan, W.S.; Yang, J.; Tan, K.S.; Er, M.H.: A digital beamsteerer for difference frequency in parametric array. IEEE Trans. Audio Speech Lang. Process., 14 (2006), 1018-1025.

[50] Shi, C.; Gan, W.S.: Grating lobe elimination in steerable parametric loudspeaker. IEEE Trans. Ultrason., Ferroelectr. Freq. Control, 58 (2011), 437-450.

[51] Shi, C.; Gan, W.S.: Product directivity models for parametric loudspeakers. J. Acoust. Soc. Am., 131 (2012), 1938-1945.

[52] Shi, C.; Gan, W.S.: Modeling the directivity of parametric loudspeaker, Int. Symp. Nonlinear Acoustics, Tokyo, Japan, 2012.

[53] Shi, C.; Nomura, H.; Kamakura, T.; Gan, W.S.: An envelope method for improving the product directivity models of the parametric loudspeaker. IEICE Technical Report, 113 (2014), 7-10.

[54] Shi, C.; Kajikawa, Y.: A convolution model for computing the far-field directivity of a parametric loudspeaker array. J. Acoust. Soc. Am., in press.

[55] Yang, J.; Gan, W.S.; Tan, K.S.; Er, M.H.: Acoustic beamforming of a parametric speaker comprising ultrasonic transducers. Sens. Actuators $A, 125$ (2005), 91-99.

[56] Harris, C.M.: Absorption of sound in air versus humidity and temperature. J. Acoust. Soc. Am., 40 (1966), 148-159.

[57] McClellan, J.H.; Parks, T.W.: A personal history of the ParksMcClellan algorithm. IEEE Signal Process. Mag., 22 (2005), 82-86.

Chuang Shi received his B. Eng. degree in Computer Science from Beijing Jiaotong University in 2005, M. Eng. degree in Microelectromechanical Systems from Tsinghua University in 2008, and Ph.D. degree in Electrical and Electronic Engineering from Nanyang Technological University in 2013. He joined Nanyang Technological University as a research associate and research fellow in 2011 and 2013, respectively. He is currently working as a postdoctoral fellow at Kansai University. He is a member of IEEE and a member of APSIPA. His research interests include parametric array loudspeaker, active noise control, acoustic beamforming, and audio classification.

Yoshinobu Kajikawa received his B. Eng. degree in 1991 and M. Eng. degree in 1993 from Kansai University in Electrical Engineering, and D. Eng. degree in 1997 from Osaka University in Communication Engineering. He worked at Fujitsu Ltd. in 1993 and engaged in research on active noise control. In 1994, he joined Kansai University, as professor. He is a senior member of IEEE and a member of APSIPA. His current research interests fall in the area of signal processing for acoustic and communication systems.

Woon-Seng Gan received his B. Eng. and Ph.D. degrees both in Electrical and Electronic Engineering from the University of Strathclyde in 1989 and 1993, respectively. He joined Nanyang Technological University as a lecturer and senior lecturer in 1993 and 1998, respectively. In 1999, he was promoted to as Associate Professor. He is an AES fellow, a senior member of IEEE, and a member of APSIPA. He has published more than 300 international refereed journal and conference papers. His current research interests include active noise control, audio signal processing, parametric loudspeaker, and spatial audio. 